\title{
Multiaxial Fatigue of Additive Manufactured Metals
}

\author{
Ali Fatemi ${ }^{1}$, Reza Molaei ${ }^{1}$, and Nam Phan $^{2}$ \\ ${ }^{1}$ Mechanical Engineering, University of Memphis, Memphis, TN, Unites States \\ ${ }^{2}$ Structures Division, US Naval Air Systems Command, Patuxent River, MD, United States
}

\begin{abstract}
Additive manufacturing (AM) has recently gained much interest from researchers and industry practitioners due to the many advantages it offers as compared to the traditional subtractive manufacturing methods. These include the ability to fabricate net shaped complex geometries, integration of multiple parts, on-demand fabrication, and efficient raw material usage, among other benefits. Some of distinguishing features of $\mathrm{AM}$ metals, as compared to traditional subtractive manufacturing methods, include surface roughness, porosity and lack of fusion defects, residual stresses due to the thermal history of the part during the fabrication process, and anisotropy of the properties. Most components made of AM processes are subjected to cyclic loads, therefore, fatigue performance is an important consideration in their usage for safety critical applications. In addition, the state of stress at fatigue critical locations are often multiaxial. Considering the fact that many of the distinguishing features of $\mathrm{AM}$ metals are directional, the subject of multiaxial fatigue presents an important study area for a better understanding of their fatigue performance. This paper presents an overview of the aforementioned issues using recent data generated using AM Ti-6Al-4V and 17-4 PH stainless steel. Specimens were made by laser-based powder bed fusion and subjected to axial, torsion, and in-phase as well as out-of-phase loadings. A variety of conditions such as surface roughness, thermo-mechanical treatment, and notch effects are included. Many aspects are considered including damage mechanisms and crack paths, cyclic deformation, fatigue crack nucleation and growth, and stress concentration effects.
\end{abstract}

\section{Introduction}

Additive Manufacturing (AM) offers a number of advantages compared to the conventional subtractive manufacturing methods such as the capability of fabricating complex geometries, which are difficult or not feasible to build using conventional methods. AM also provides the ability to design functionally graded materials as well as eliminating some jointing and assembly issues which in addition to increasing cost, often limit the performance of parts made by the traditional fabrication methods. Much less or in some

\footnotetext{
${ }^{1}$ Corresponding author: afatemi@memphis.edu
} 
cases no material waste as compared to the conventional subtractive manufacturing techniques, efficient production, capability of on-site fabrication of replacement parts, and the ability of producing parts in small numbers are among other benefits offered by the AM techniques.

In order to use AM parts in critical applications, such as those in aerospace and biomedical industries, cyclic properties and fatigue performance are of utmost important considerations as such parts are commonly subjected to cyclic loads in their operational environments. In addition, biaxial and multiaxial stresses are common for many components, even under nominal uniaxial loading conditions where the stress state can be multiaxial due to the complexities in the geometry such as notches, or presence of multidirectional residual stresses [1]. Such effects are more pronounced in AM, where geometry complexities result in stress concentrations, multidirectional residual stresses from the fabrication process are inevitable, and produced defects are typically directional resulting in anisotropy.

Defects size, shape, location, density, and orientations are significantly affected by the utilized processing parameters, build direction, and even the number of samples per build. These defects, most commonly Lack of Fusion (LOF) and porosity, significantly affect fatigue performance of the fabricated parts. Detrimental effect of these defects on fatigue strength varies with their alignment with respect to the complex multiaxial stress state.

Despite the aforementioned challenges, there is a very small number of studies in the literature with respect to the multiaxial fatigue behavior of AM metallic materials [2-6]. In this paper, after a brief overview on different fabrication and post fabrication processes, the effect of process parameters and thermal histories during fabrication as well as subsequent post treatments on the resulting microstructures and defect structure are discussed. Importance of damage mechanism and cracking behavior under different stress states and their effects on cyclic deformation and fatigue behavior are discussed next. Data correlations under different stress states and the effect of stress concentrations are also discussed. Finally, a brief discussion of some of the other important issues not considered in this paper is presented and an outlook for future research is provided.

\section{Fabrication and Post Process Treatments}

Depending on the heat source of the AM systems, they can be classified as laser-based, electron beam-based, and arc-based systems. Material in the AM process can be supplied by either powder or wire feed. Among different fabrication systems, Powder Bed Fusion (PBF) and Directed Energy Deposition (DED) are the most commonly used AM processes. In PBF, the powder is melted via laser or electron beam over the previous layer, while in DED the powder or wire infuse simultaneously with the energy source which could be laser or electron beam.

As compared to DED, PBF has the capability to fabricate more complex geometries with high geometrical accuracies as well as better surface finish. This ability is considered to be the major advantage of this process, as compared to the DED methodology [7]. However, DED is faster in fabrication, and is also capable to fabricate larger parts as compared to PBF. Smaller particles and thinner build layers as well as slower scan strategies employed by laser-based PBF machines result in finer features, better surface finishes, and higher build tolerances as compared to the electron-beam PBF machines.

Due to the many synergistic factors affecting fatigue behavior of AM parts, predicting their fatigue behavior is still very challenging. These include microstructural aspects such as grain structure and defect characteristics, surface roughness, residual stresses, and build orientation or anisotropy. Several process parameters such as energy source input, scanning 
speed, hatch spacing, and layer thickness are among the most important factors in controlling these aspects, and consequently mechanical performance of the fabricated parts.

Surface roughness of the fabricated parts is one of the major factors that needs to be considered in investigating the fatigue behavior of AM parts since fatigue cracks most often start from the surface. The rough surface is attributed to the stair-stepping effect and also depends on the curvature of the surface, deposited layer thickness, partially melted particles, and presence of material defects [8]. In some applications a rougher surface finish may be favourable over a smooth surface finish such as for orthopaedic implants. But in most cases, due to its detrimental effects on durability of the AM parts subjected to cyclic loads, post-manufacturing processes such as surface machining are often desirable to reduce the surface roughness. However, as mentioned earlier, one of the major benefits of the AM technique is the capability of fabricating complex geometry parts. Therefore, machining of these complex geometries for enhanced surface finish subsequent to AM would be difficult, if not impossible.

Thermal gradients, energy density, and most importantly fast solidification during the AM processes result in residual stresses. Detrimental residual stresses may be eliminated or reduced with applying appropriate fabrication process parameters and build layer orientations, or with applying post-fabrication heat treatment processes. There is a relatively large number of studies in the literature indicating the effects of different heat treatments in enhancing the fatigue behavior of AM materials. These post-fabrication treatments if done at proper temperatures and durations, may substantially reduce the residual stresses of the fabricated parts, improve the ductility via making changes in the microstructures, as well as minimizing the anisotropy of AM parts. Among different treatments, Hot Isostatic Pressing (HIP) as a thermo-mechanical process has been found to be most effective. This treatment not only can change the microstructure and relax or reduce residual stresses, it will also densify the material with shrinking and closing of the internal defects [5].

\section{Microstructure and Defect Structure}

Similar to the conventionally fabricated alloys, microstructural characteristics of the AM parts are significant contributors to their mechanical behavior. Depending on the material, AM system used, process parameters, and experienced thermal history during fabrication, the resultant microstructure could notably vary. Different build orientations as well as the number of samples per build could also affect the heat transfer between different layers and cause substantially different cooling rates and, thus, microstructures. As an example, the typical microstructures of Ti-6Al-4V alloy resulting from different AM processes and before and after undergoing subsequent heat treatments (HT), along with the conventional wrought microstructure are shown in Fig. 1. As can be seen, not only the microstructure may significantly change after undergoing different heat treatment processes, but it could also vary between different AM systems.

The extent by which the fatigue behavior is affected by the AM defects mainly depends on the microstructure and ductility present in the material [9]. Entrapped gas pores which are mostly spherical, and LOF defects which have irregular shapes have been reported to be the most responsible causes of fatigue failures in AM fabricated parts. Based on the processing parameters, scan strategies, and build orientations, defects may be formed and distributed in specific directions with implications on multiaxial fatigue performance. Entrapped gas pores form at excessive energy densities, and the LOF defects occur at insufficient energy densities. Choosing proper process parameters may result in fabricating parts without significant LOFs. Examples of these defects are shown in Fig. 2(a). Even after 
applying the HIP process some defects can remain which could be fatigue crack initiators. An example of these defects is shown in Fig. 2(b).
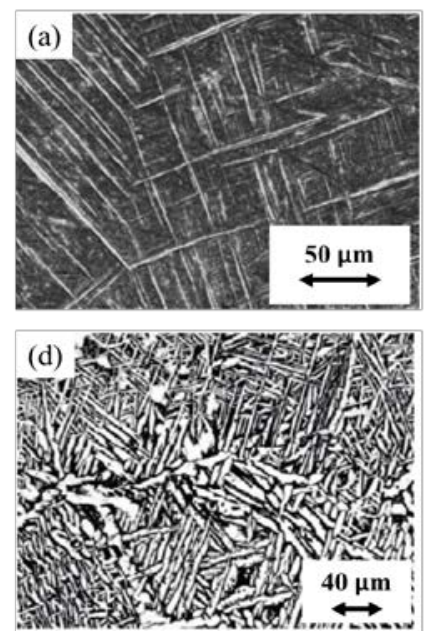
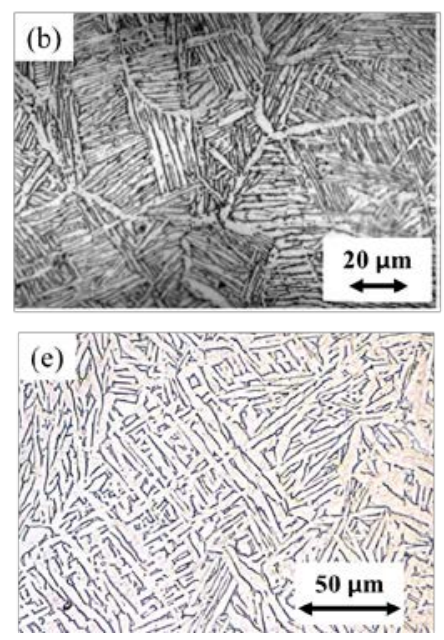
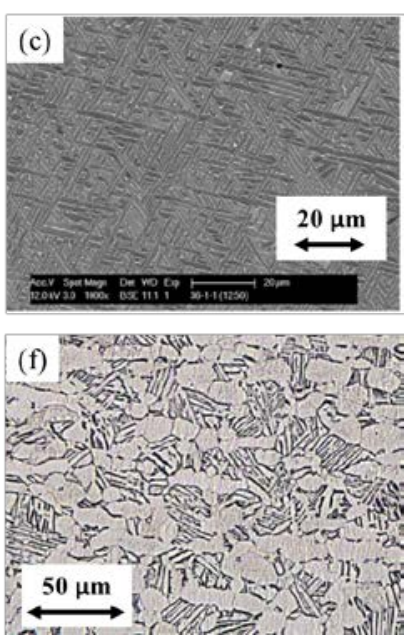

Fig. 1. Microstructure of AM and wrought Ti-6Al-4V alloy. (a) Acicular martensite of as-built L-PBF process [10], (b) coarse Widmanstätten/basket weave of as-built E-beam PBF process [11], (c) fine Widmanstätten/basket weave of L-DED process [12], (d) duplex structure of L-PBF process after HT [13], (e) elongated $\alpha$ laths in $\alpha-\beta$ dual-phase of L-PBF process after HIP [14], and (f) equiaxed $\alpha-\beta$ dual-phase of wrought [14].

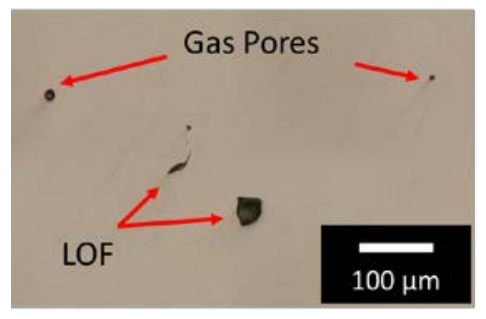

(a)

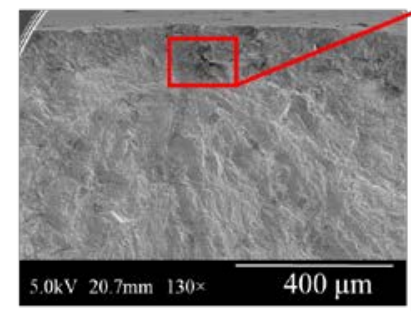

(b)

Fig. 2. (a) Entrapped gas pores and LOF defects in L-PBF Ti-6Al-4V. (b) Fatigue crack initiation from un-melted particles even after the HIP process.

Machine-to-machine process parameter variabilities can affect defect formation of the AM materials, even for the same AM method. Fig. 3(a) shows number of defects vs. defect largest length for AM Ti-6Al-4V specimens fabricated with two different AM L-PBF machines with different process parameters (different energy densities). As can be seen, the density and size of the defects generated between the two machines are very different. Moreover, it is evident that when the initial defects are large, the HIP process is not as effective in closing or shrinking such defects. As shown in Fig. 3(b), although there is a randomness for the defects to occur in $\mathrm{x}-\mathrm{z}$ and $\mathrm{y}-\mathrm{z}$ planes for the specimens built in $\mathrm{z}$ direction, larger defects in terms of the size and area occur in the plane perpendicular to the build orientation (x-y plane) [15]. Again, such non-uniform variation can result in anisotropy of fatigue performance with implications on multiaxial fatigue behavior with respect to the applied stress direction. 


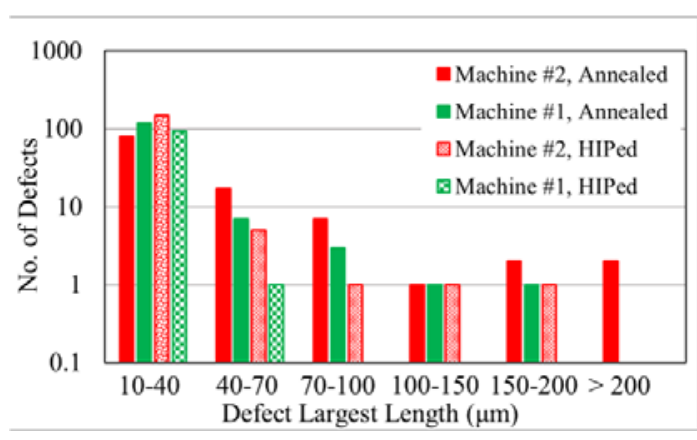

(a)

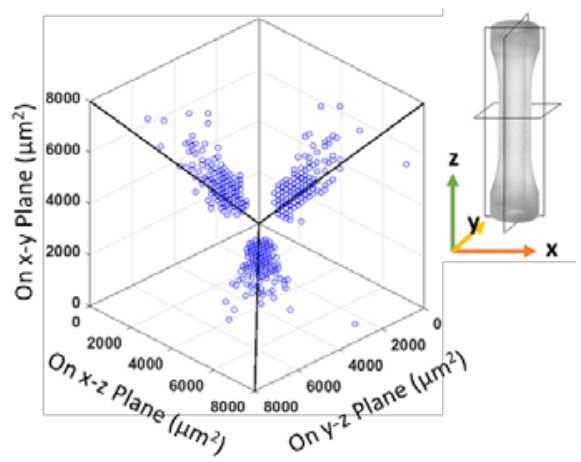

(b)

Fig. 3. (a) Comparison of defect numbers and size densities between Ti-6Al-4V specimens fabricated with two different types of L-PBF machines with or without HIP. (b) Defect projection area on different planes for Ti-6Al-4V [15].

\section{Damage Mechanisms and Cracking Behavior}

Knowing the failure mechanism and cracking behavior of the material under cyclic loading is essential to performing accurate fatigue analysis. Microstructure and ductility, as well as the stress state and magnitude affect the mechanism and cracking behavior. For AM metals defects and anisotropy which may be unavoidable are also other significant influencing factors [4].

While fatigue cracks in wrought metals typically initiate from microscopic regions of localized plastic deformation and grain boundaries resulting from dislocations and slip systems, defects, both internal and surface, have been shown to be the biggest contributors to fatigue failure initiation of AM metals. Stress concentration at the vicinity of these defects is a major factor and its value depends on the size, shape, and location of the defect.

It is well known that naturally occurring fatigue cracks originating from slip systems are controlled by a shearing process and, therefore, occur on planes of maximum shear. These mode-II cracks then could grow in the same orientation, or branch to mode-I. However, this may not be the case for AM metals, where defects and anisotropy of the microstructure could substantially affect the cracking behavior.

Metal fatigue damage process mainly includes nucleation and growth of micro-cracks up to a length of about a few grains on the order of one or two hundred microns, followed by small crack growth (typically less than $2 \mathrm{~mm}$ ), and then long crack growth $(>2 \mathrm{~mm})$ [16]. Since defects are present in the AM fabricated parts, the first step of crack nucleation may be non-existent. Therefore, fatigue life may be composed of small crack and possibly long crack growth processes.

Fatigue crack length vs. normalized number of cycles for two different metals are shown in Fig. 4(a) for specimens subjected to in-phase axial-torsion loads. Although no significant difference between the crack growth behavior of the AM and wrought 17-4 PH alloys is observed, many more cracks in various sizes were observed for the wrought alloy, while fewer cracks with smaller sizes were evident on the surface of the AM alloy. In addition to the difference in ductility, this could be due to the presence of internal defects in the AM metal and their coalescence. As can be seen from Fig. 4(a), for both AM Ti-6Al-4V and 17-4 PH alloys, as fatigue life increases crack growth period becomes a smaller fraction of total life, which is consistent with that of the conventional wrought metal.

Depending on the microstructure, residual stresses, and defects, fabricated AM parts in the as-built condition typically behave in a brittle manner with a tensile failure mode. 
Application of different post-fabrication stress relieving or heat treatment processes as well as surface machining could improve the ductility of the fabricated parts and, therefore, may change the damage mechanism and cracking to a more ductile behavior and shear failure mode. For example, as can be seen in Figs. 4(b) and 4(c) for machined surface conditions, although annealing at $700^{\circ} \mathrm{C}$ for 1 hour still results in tensile failure, application of Hot Isostatic Pressing (HIP) on Ti-6Al-4V specimens can alter the failure mechanism of the material and transform the tensile failure to shear. This sensitivity to the post-fabrication processes could be considered as an opportunity to tailor properties of AM fabricated parts to specific applications.

The rough surface and surface defect networks of AM fabricated parts could play an important role in governing crack initiation and growth directions. For example, in [17] it was shown that for AM 17-4 PH specimens, after surface machining the failure initiation plane was along the maximum shear plane orientation, while the weak build plane and surface defect networks dictated cracking orientation when the rough surface was present. Similar behavior can be seen in Fig. 4(c) for the HIPed Ti-6Al-4V alloy with as-built surface condition, where the rough surface controls the failure mechanism and results in tensile failure. Capturing the correct damage mechanism is necessary for selecting the appropriate damage parameter for data correlations and fatigue life analysis under multiaxial loading. This is difficult to do under uniaxial loading, due to the existence of four maximum shear planes and their intersections.

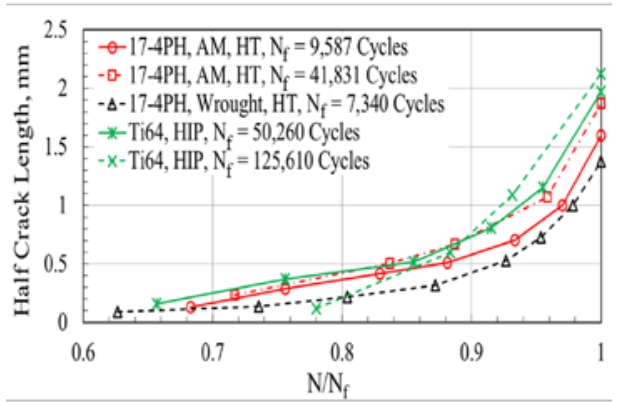

(a)

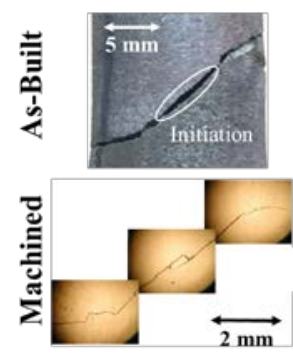

(b)

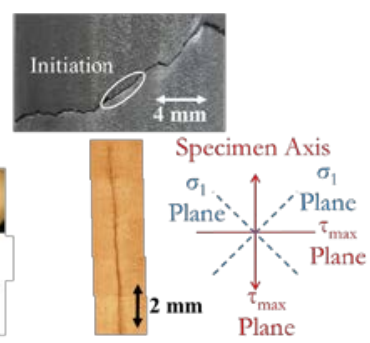

(c)

Fig. 4. (a) Small fatigue crack growth for AM HIPed Ti-6Al-4V and 17-4 PH heat treated AM and wrought alloys under in-phase axial-torsion loading with different lives. Representative crack orientations for AM Ti-6Al-4V alloy under torsion loading with as-built and machined surface conditions for (b) annealed, and (c) HIPed conditions. Solid red lines indicate the maximum shear planes and blue dashed lines indicate the maximum principal planes.

\section{Cyclic Deformation}

The response of a material under cyclic loading can vastly differ from its monotonic response due to phenomenon such as cyclic softening or cyclic hardening. In addition, under multiaxial stresses some materials experience additional strain hardening due to the non-proportionality of the cyclic load. The amount of non-proportional hardening depends on the material microstructure, stress amplitude, and degree of non-proportionality between the stress components.

Due to different process parameters and also distinct thermal histories during the AM processes, different monotonic and/or cyclic deformation behaviors may occur. For example, while Sterling et al. [18] did not observe notable cyclic softening or hardening for DED Ti-6Al-4V specimens under axial loading, Fatemi et al. [2] reported some softening of annealed L-PBF Ti-6Al-4V specimens under torsion loading. As can be seen in Fig. 5, although the monotonic properties of the wrought and AM materials are similar, their cyclic 
behaviors are different. As seen, the amount of cyclic softening for the AM HIPed material is about half of that for the wrought material. Even after undergoing the same HIP treatment, the AM specimens fabricated in different orientations may still show distinct cyclic responses due to the remaining directional defects the resulting anisotropy in the microstructure.

Therefore, depending on the AM process and parameters used, both monotonic as well as cyclic deformation behaviors can significantly change and also differ from each other. Proper fatigue analysis where plastic deformation is present requires characterization of the cyclic deformation behavior. Under multiaxial stress conditions, assessing the degree of stress non-proportionality and its effect on non-proportional hardening is also required. However, regardless of the material failure mode, von-Mises equivalent stress and strain can correlate the multiaxial cyclic deformation data under different stress states reasonably well in most cases, such as that shown in Fig. 5.

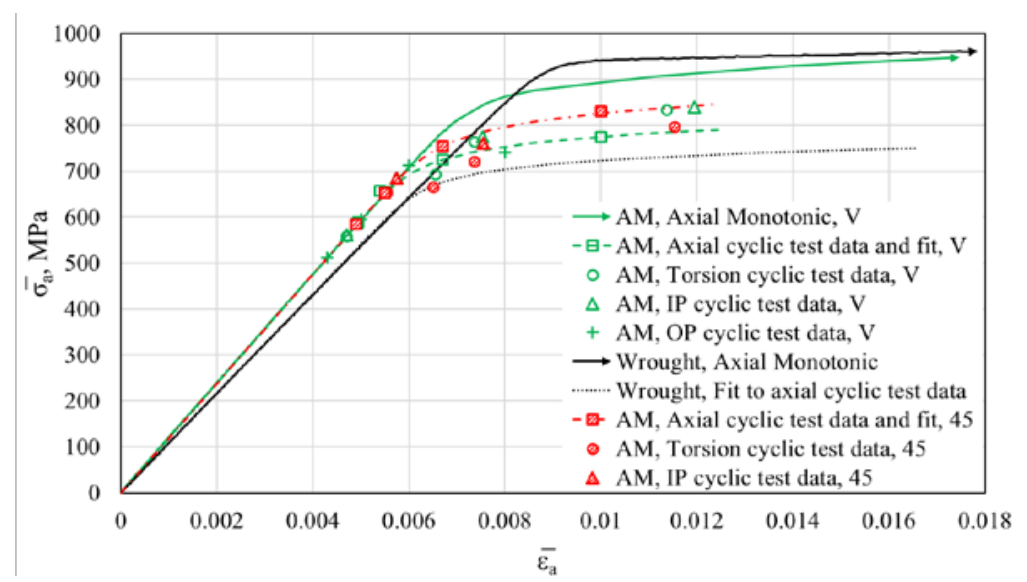

Fig. 5. Superimposed monotonic and mid-life von-Mises equivalent stress and strain data and curves for wrought and vertical and diagonal (at $45^{\circ}$ ) built L-PBF HIPed Ti-6Al-4V machined surface specimens [5].

\section{Fatigue Failure Criteria and Life Estimation}

Classical yield criteria such as von-Mises equivalent stress or strain and maximum principal stress or strain are often used for multiaxial fatigue analysis. Although these approaches may work for simple loading conditions such as proportional loadings, they lack a general robustness when the loading conditions become more complex, such as non-proportional loading often experienced under service loading conditions [19, 20].

Critical plane-based approaches have the capability of reflecting the damage mechanisms of the material and can, therefore, not only provide fatigue life estimation under different stress states, but also predict the highest damage (i.e. critical) plane orientation. Consistent with the damage mechanism of the material as discussed in Section 4, appropriate critical plane-based damage parameters can be chosen. For example, the Fatemi-Socie (FS) fatigue damage parameter [21] can be chosen as a shear-based critical plane approach when the failure mechanism is shear, and the Smith-Watson-Topper [22] as a tensile-based critical plane approach when failure mechanism is tensile. As stated earlier, the failure mechanism in AM metals depends on many factors and even for a given alloy the failure mechanism can differ depending on the microstructure and defects, which in turn depend on the fabrication process parameters and post process treatment(s) used. 
As an illustration, multiaxial data correlations for L-PBF HIPed Ti-6Al-4V alloy based on the von-Mises and FS critical plane approaches are shown in Fig. 6. As can be seen in Fig 6(a), von-Mises equivalent strain criterion does not correlate either the in-phase or the out-of-phase fatigue test data with the axial and torsion data. The critical plane-based damage parameter on the other hand correlate all the multiaxial fatigue data including outof-phase data, as can be seen in Fig. 6(b). This is consistent with the shear damage mechanism observed for this material in [5] as discussed in Section 4.

However, as discussed previously, the internal and surface defects may govern the cracking behaviors and, hence, could affect the choice of the damage parameter and, consequently, fatigue life estimations. It was shown in [4] that the failure mechanism of the L-PBF annealed Ti-6Al-4V alloy is significantly affected by defects (machine \#2 in Fig. 3(a)), resulting in tensile failure in both the as-built and machined surface conditions (Fig. 4(b)). Consequently, it was shown that a criterion such as maximum principal stress correlates the data well.

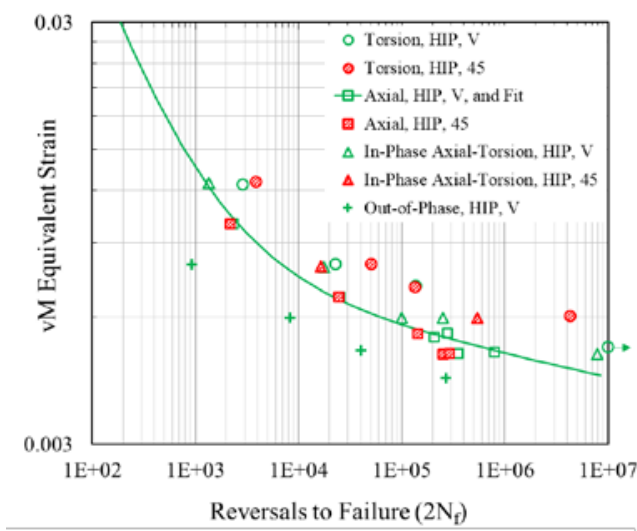

(a)

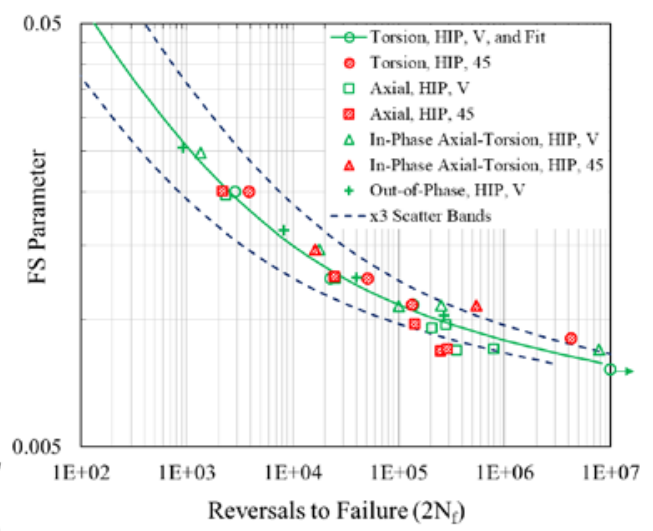

(b)

Fig. 6. Correlation of multiaxial fatigue test data of L-PBF machined surface HIPed Ti-6Al-4V alloy based on (a) von-Mises equivalent strain criterion and, (b) Fatemi-Socie damage parameter [5].

Due to the dominance of defects for AM metals, both internal and surface, their effect on fatigue life estimations may have to be incorporated explicitly. Therefore, when defects dominate fatigue behavior, using nominal stress and/or strain may not be sufficient to characterize fatigue performance and some additional information such as defect size or area may need to be included in damage quantification. In this regard, fracture mechanicsbased parameters such as Stress Intensity Factor (SIF) may also need to be considered for the analysis.

Zhang and Fatemi [23] used such a method to account for the stress concentration of the surface defects for multiaxial fatigue life estimations. The surface roughness was treated as surface cracks with considering the sum of maximum surface valley, $R_{v}$, as the total crack depth, and defect induced crack length, $a_{0}$. By assuming a semi-circular surface crack geometry, the SIF was calculated from:

$$
K=0.723 \sigma \sqrt{\pi\left(R_{w}+a_{0}\right)}
$$

Fatigue life estimations were then performed based on integrating the Paris equation: 


$$
N_{f}=\int_{R_{V}}^{a_{c}} \frac{d(a)}{A(\Delta K)^{n}}
$$

where $a_{c}$ is the failure crack length which was taken as $5 \mathrm{~mm}$. Fatigue life correlations as well as predictions based on the surface roughness parameter for as-built surface annealed and HIPed Ti-6Al-4V specimens under different multiaxial loading conditions are shown in Fig. 7. Such methods have the potential to correlate multiaxial fatigue data from conditions resulting in different defect sizes and/or surface roughnesses.

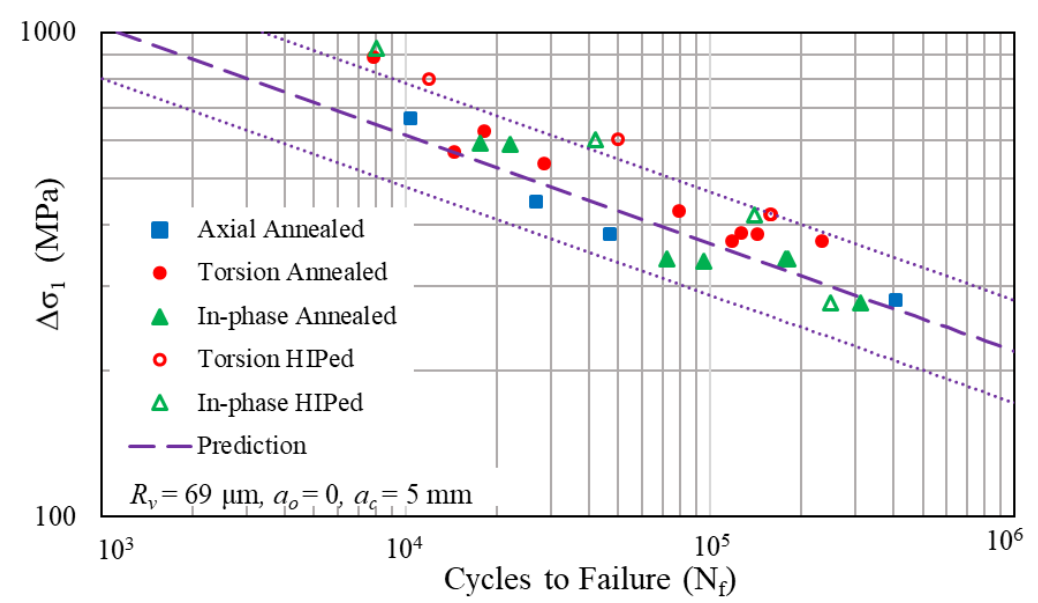

Fig. 7. Fatigue life predictions based on surface roughness parameter for as-built surface Ti-6Al-4V annealed and HIPed specimens with scatter band of \pm 3 [23].

\section{Stress Concentration Effect on Fatigue Life}

As discussed in Section 1, the capability of manufacturing complex geometries is one of the main advantages offers by AM in which numerous stress concentrations may be present, such as in lattice structures. Therefore, it is necessary to evaluate and characterize the cyclic behavior of AM metals in the presence of notches and stress concentrations. In addition, the rough surface and un-melted particle clusters on the surface of the AM fabricated parts can also act as stress concentrations and significantly affect the behavior. As a result, how such inherent stress concentrations to the AM process interact with stress concentrations due to component geometry in affecting fatigue life also has to be considered.

For conventionally fabricated ductile behaving metals it has been shown that while the naturally initiated crack at the notch root initiates and grows on the order of a few grains on the maximum shear plane, it tends to turn to the maximum tensile plane for the rest of its development period [24]. However, presence of the surface and/or internal defects in AM metals may affect this cracking behavior.

Cracking behavior of two AM fabricated metals, Ti-6Al-4V and 17-4 PH alloys, with different heat treatments and different surface conditions were studied in presence of a transverse hole in tubular specimens in [17]. It was found that although the cracking behavior for the machined surface condition could be similar to the conventionally fabricated wrought metal, it may differ when the as-built rough surface is present. In addition, it was observed that regardless of the surface roughness condition, cracks always initiated from the highest stressed location at the notch. This indicates that when the mechanically induced stress concentrations are present, depending on the severity of the 
notch stress concentration, the presence of defects may no longer play an important role on fatigue life.

Although the crack growth direction for the two aforementioned AM metal alloys with the transverse hole was along the maximum principal plane in mode-I for both as-built and machined surface conditions, the initiation orientations were different, as shown in Fig. 8(a). While the initiation orientations were along or near the maximum shear planes for the machined surface 17-4 PH, cracks mainly initiated and grew along the maximum principal plane orientations when the rough surface was not machined. The transition from mode-II to mode-I for the machined surface condition is mainly due to the frictional effects between the crack faces which could restrict the growth in the same orientation and result in transition to mode-I. However, friction caused by the rough surface and un-melted particles of the as-built surface specimens may also affect this roughness induced closure mechanism. This is similar to the findings for the as-built surface unnotched specimens, where the rough surface substantially controls the failure mechanism.

As can be seen in Fig. 8(b) for the small number of in-phase axial-torsion fatigue test results of the Ti-6Al-4V and 17-4 PH alloys, FS damage parameter correlates the notched data with the unnotched data based on the same local damage value of the notched and unnotched specimens. In order to calculate the local damage of the notched specimens, after finding the critical locations at the notch root using FEA and considering the stress concentrations and gradient effects, the local strain and stress values at the notch critical locations were calculated. Notch stress gradient effects are important considerations in fatigue analysis of notched components. There are several methods to consider the effect of the stress gradient at the notch root, for example, Theory of Critical Distance (TCD) [25].
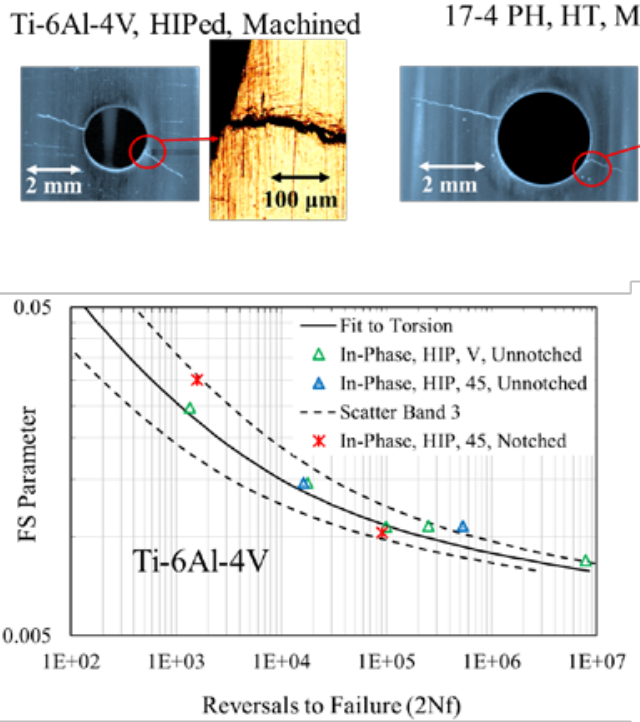

17-4 PH, HT, Machined

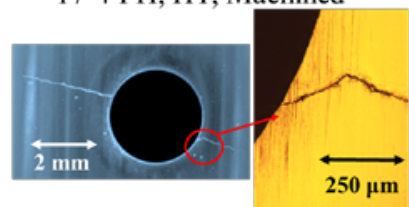

(a)

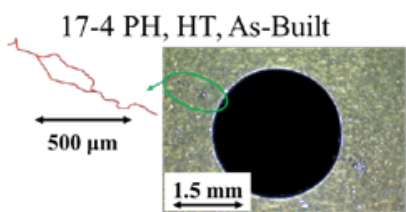

$\stackrel{1.5 \mathrm{~mm}}{\longrightarrow}$

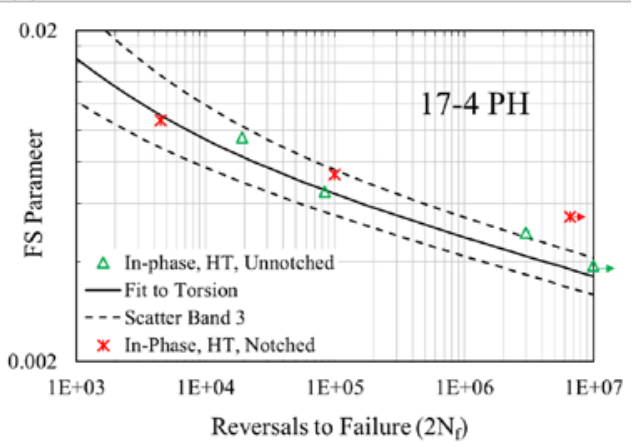

(b)

Fig. 8. (a) Representatives for the failure crack orientations of notched specimens of different metals and surface conditions [17]. (b) Correlation of the axial-torsion unnotched and notched fatigue test results of L-PBF machined surface HIPed Ti-6Al-4V, and heat treated at 1025 17-4 PH alloys based on FS critical plane-based damage parameter. 


\section{Summary and Outlook for Future Research}

Additive Manufacturing (AM) offers a number of significant advantages compared to the conventional subtractive manufacturing methods. In order to use AM parts in most critical applications, however, cyclic properties and fatigue performance are of utmost important considerations. Multiaxial fatigue is of high importance not only because of the presence of multiaxial loads, but due to other features which are prevalent in AM components and structures. These include multiaxial stress states at fatigue critical locations due to the geometrical complexities in AM structures even under uniaxial loading, multidirectional residual stresses from the inevitable fabrication process, and directionality of the microstructure and defects which may result in anisotropic behaviour.

In this paper, first a brief overview on different fabrication and post fabrication processes was provided. The effects of process parameters and thermal histories during fabrication as well as subsequent post treatments on the resulting microstructures and defect structures were then reviewed. Importance of damage mechanism and cracking behavior under different stress states and their effects on cyclic deformation and fatigue behavior were discussed next, followed by fatigue data correlations under different stress states and the effect of stress concentrations.

While fatigue cracks in wrought metals typically initiate from microscopic or macroscopic regions of localized plastic deformation, defects, both internal and surface, have been shown to be the biggest contributors to fatigue failure initiation of AM metals. Fatigue failure mechanism in AM metals varies, even for a given metal and identical fabrication method, depending on the process parameters and post process treatments. Capturing the correct damage mechanism is necessary for selecting the appropriate damage parameter for data correlations and fatigue life analysis under multiaxial loading.

Proper fatigue analysis where plastic deformation is present requires characterization of the cyclic deformation behavior. Depending on the AM process and parameters used, both monotonic as well as cyclic deformation behaviors can significantly change and also differ from each other. AM specimens fabricated in different orientations may show distinct cyclic responses due to the directional defects and the resulting anisotropy in the microstructure.

Internal and surface defects may govern the damage mechanism and cracking behavior and, hence, affect the choice of the damage parameter to be used. Critical plane-based damage parameters can correlate multiaxial fatigue data, consistent with the damage mechanism observed for the material. However, due to the dominance of defects for AM metals, both internal and surface, their effect on fatigue life estimations may have to be incorporated explicitly. In this case, fracture mechanics-based parameters can be considered for fatigue life analysis.

Presence of surface and/or internal defects in AM metals can also affect notched fatigue behavior. The rough surface and un-melted particle clustered on the surface of the AM fabricated parts act as stress concentrations and can significantly affect the behavior. As a result, how such inherent stress concentrations to the AM process interact with stress concentrations due to component geometry in affecting fatigue life has to be considered.

Residual stresses are typically large and multi-directional in AM fabricated parts. Selecting proper fabrication process parameters, making appropriate choices in build orientation and number of parts per build, as well as post fabrication heat treatment processes can significantly affect and decrease residual stresses. If they still remain during the cyclic loading process, their effect needs to be considered in cyclic and fatigue life analysis using appropriate measurement and modelling techniques.

Not only the size, but shape, orientation, location, and distribution of defects could can affect the fatigue behaviors. Due to the large number of parameters affecting the defect 
formations in AM processes, it may not be practical or efficient to quantify their effects on fatigue performance by testing. Hence, development of effective non-destructive techniques to characterize defects and reliable statistical models to quantitatively measure their effect on fatigue behaviour, including under multiaxial stress states, are highly desirable.

Different AM systems with different processing parameters could result in different roughness profiles. Since one of the main advantages of the AM technique is the ability of fabricating complex geometries, removing the rough surface in complex geometry parts may be difficult, if not impossible. The rough surface and surface defects and micro-cracks are often fatigue crack initiation locations. Quantitative models are needed to account for the effect of these surface discontinuities on fatigue performance in general, and on multiaxial fatigue performance in particular.

Fatigue crack growth often represents a notable portion of the fatigue life [24]. This could be more important for AM metals, where defects are present from the time of the manufacture. Small crack growth mechanism may be in mode-II or mixed-mode under multiaxial stress states. Such effects may be more pronounced for AM metals where directionality of the defects and anisotropy in the microstructures can cause retardation or acceleration of crack growth, particularly in presence of multi-directional residual stresses.

In spite of service load application being typically variable amplitude in nature, nearly all the studies of AM metals are with small specimens subjected to constant amplitude loading in laboratory air environment. When the loading is variable amplitude, load sequence and their interactions under multiaxial stresses can play important roles in the fatigue damage and accumulation process. In addition, the effect of common service operating environments such as corrosion and elevated temperatures, as well as size effects to facilitate extrapolation of data from small specimens to larger components, also need to be evaluated.

Finally, qualification and certification standards and procedures for AM metals and parts in safety critical applications are currently at the very initial stages of development. Such standards and procedures could not practically rely on large numbers of data, typically generated for conventional metals and processes, due to relatively small batch sizes and long build times in AM processes [26]. Therefore, unique qualification and certification techniques tailored to such processes need to be developed.

\section{References}

1. A. Fatemi, N. Shamsaei, "Multiaxial fatigue: An overview and some approximation models for life estimation, Int. J. Fatigue, 33 (2011).

2. A. Fatemi, R. Molaei, S. Sharifimehr, N. Shamsaei, N. Phan, "Torsional fatigue behavior of wrought and additive manufactured Ti-6Al-4V by powder bed fusion including surface finish effect, Int. J. Fatigue, 99 (2017).

3. A. Fatemi, R. Molaei, S. Sharifimehr, N. Shamsaei, N. Phan, "Torsional fatigue behaviour of wrought and selective laser melted Ti-6Al-4V including as-built and machined surface finish effects, 17th Aust. Int. Aero. Cong.: AIAC, (2017).

4. A. Fatemi, R. Molaei, S. Sharifimehr, N. Phan, N. Shamsaei, "Multiaxial fatigue behavior of wrought and additive manufactured Ti-6Al-4V including surface finish effect, Int. J. Fatigue, 100 (2017).

5. R. Molaei, A. Fatemi, N. Phan, "Significance of hot isostatic pressing (HIP) on multiaxial deformation and fatigue behaviors of additive manufactured Ti-6Al-4V including build orientation and surface roughness effects, Int. J. Fatigue, 117 (2018).

6. P. Carrion, A. Imandoust, J. Simsiriwong, N. Shamsaei, "Effects of layer orientation on the multiaxial fatigue behavior of additively manufactured Ti-6Al-4V, 29th Annu. Int. S. Freeform Fab. Symp. Proc., (TX, 2018). 
7. T. DebRoy, H. Wei, J. Zuback, T. Mukherjee, J. Elmer, J. Milewski, et al., "Additive manufacturing of metallic components - proc., struct. and prop., Prog. in Mat. Sci., 92 (2018).

8. P. Li, D. Warner, A. Fatemi, N. Phan, "Critical assessment of the fatigue performance of additively manufactured $\mathrm{Ti}-6 \mathrm{Al}-4 \mathrm{~V}$ and perspective for future research, Int. J. Fatigue, 85 (2016).

9. A. Fatemi, R. Molaei, J. Simsiriwong, N. Sanaei, J. Pegues, B. Torries, et al., "Fatigue behavior of additive manufactured materials: An overview of some recent experimental data for Ti-6Al-4V considering various processing and loading direction effects, Fatigue \& Fract. Eng. Mat. \& Struct., doi.org/10.1111/ffe.13000 (2019).

10. D. K. Do, P. Li, "The effect of laser energy input on the microstructure, physical and mechanical properties of Ti-6Al-4V alloys by selective laser melting, Virt. Phys. Prot., 11 (2016).

11. H. Rafi, N. Karthik, H. Gong, T. L. Starr, B. E. Stucker, "Microstructures and mechanical properties of Ti-6Al-4V parts fabricated by selective laser melting and electron beam melting, J. Mat. Eng. Perf., 22 (2013).

12. B. Baufeld, E. Brandl, O. Van der Biest, "Wire based additive layer manufacturing: Comparison of microstructure and mechanical properties of Ti-6Al-4V components fabricated by laser-beam deposition and shaped metal deposition, J. Mat. Proc. Tech., 211 (2011).

13. Z. Fan, H. Feng, "Study on selective laser melting and heat treatment of Ti-6Al-4V alloy, Res. Phys., 10 (2018).

14. T. M. Mower, M. J. Long, "Mechanical behavior of additive manufactured, powderbed laser-fused materials, Mat. Sci. Eng.: A, 651 (2016).

15. N. Sanaei, A. Fatemi, "Analysis of variability in metal additive manufacturing defect characteristics, J. AM, (Under Preparation, 2019).

16. N. Shamsaei, A. Fatemi, "Small fatigue crack growth under multiaxial stresses, In. J. Fatigue, 58 (2014).

17. R. Molaei, A. Fatemi, "Crack paths in additive manufactured metallic materials subjected to multiaxial cyclic loads including surface roughness, HIP, and notch effects, Int. J. Fatigue, (2019).

18. A. Sterling, N. Shamsaei, B. Torries, S. M. Thompson, "Fatigue behaviour of additively manufactured Ti-6Al-4V, Proc. Eng., 133 (2015).

19. N. Gates, A. Fatemi, "Multiaxial variable amplitude fatigue life analysis including notch effects, Int. J. Fatigue, 91 (2016).

20. A. Fatemi, D. Socie, "Multiaxial fatigue: Damage mechanisms and life predictions, Adv. Fatigue Sci. Tech., (1989).

21. A. Fatemi, D. F. Socie, "A Critical plane approach to multiaxial fatigue damage including out-of-phase loading, Fatigue \& Fract. Eng. Mat. \& Struct., 11 (1988).

22. K. Smith, P. Watson, T. Topper, "A stress-strain parameter for the fatigue of metals, J. Mat. JMSLA, 5 (1970).

23. J. Zhang, A. Fatemi, "Surface roughness effect on multiaxial fatigue behavior of additive manufactured metals and its modeling, Theo. App. Fract. Mech., (Under Review, 2019).

24. N. Gates, A. Fatemi, "Notched fatigue behavior and stress analysis under multiaxial states of stress, Int. J. Fatigue, 67 (2014).

25. L. Susmel, "Multiaxial notch fatigue, Woodhead Publishing, (2009).

26. R. Molaei, A. Fatemi, "Fatigue design with additive manufactured metals: issues to consider and perspective for future research, Proc. Eng., 213 (2018). 